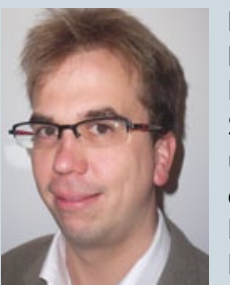

Prof. Dr. med.

Helge Frieling

Klinik für Psychiatrie,

Sozialpsychiatrie

und Psychotherapie

der Medizinischen

Hochschule

Hannover

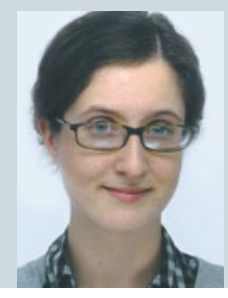

Alexandra

Kleimann

Ärztin an der Klinik

für Psychiatrie, Sozial-

psychiatrie und

Psychotherapie der

Medizinischen

Hochschule Hannover

\title{
Neue Studien zur Depression
}

\author{
Von der Pathophysiologie bis zur Telemedizin spannen die Referate den Bogen zum Thema \\ Depression. So manches zeigt sich da anders als gedacht: Die Suizidalität nimmt womöglich \\ nicht mit Beginn einer Antidepressivatherapie zu, Kreatin könnte tatsächlich als Verstärker \\ von Antidepressiva wirksam sein und eine Persönlichkeitsstörung lässt sich oft schon in \\ einer akuten depressiven Phase feststellen.
}

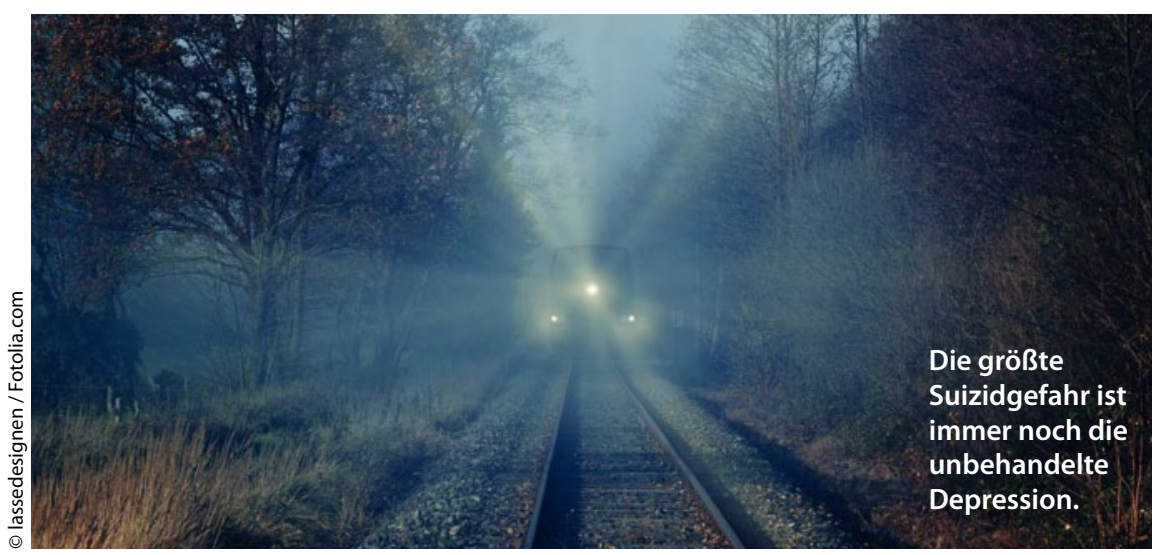

\section{Suizidgedanken durch Antidepressiva bei Jugendlichen - ein Mythos?}

Seit dem Warnhinweis der amerikanischen Gesundheitsbehörde FDA 2004 über die Erhöhung des Suizidrisikos durch Antidepressiva bei Jugendlichen herrscht Unsicherheit im Umgang mit selektiven Serotonin-Wiederaufnahmehemmern (SSRI) und anderen Antidepressiva. Nach acht Jahren wendete sich eine Studie erneut dem wichtigen Thema zu.

ie Autoren führten eine Metaanalyse von 41 placebokontrollierten Studien mit Fluoxetin und Venlafaxin durch. Primärer Endpunkt der Analyse waren Suizidgedanken, welche mittels eines Items der Hamilton-Skala und der revidierten Children's Depression Rating Sca- le (CDRS-R) gemessen wurden. Insgesamt flossen somit die teilweise unveröffentlichten Daten von 9.000 jugendlichen, erwachsenen und geriatrischen Patienten in die Auswertung ein. Danach nahm die Suizidalität von erwachsenen Patienten während der Therapie mit Fluoxetin oder
Venlafaxin ab. Je deutlicher sich die Depression besserte, ob in der Placebo- oder in der Behandlungsgruppe, desto weniger suizidal zeigten sich die Patienten. Bei Jugendlichen konnte dieser Effekt nicht gezeigt werden, ihre lebensmüden Gedanken oder Absichten wurden während der medikamentösen Therapie oder nach Besserung der Depression nicht vermindert. Die gefürchtete Zunahme von Suizidalität unter der medikamentösen Therapie war aber in keiner Gruppe zu beobachten.

Kommentar: Auch wenn es für eine Entwarnung sicher zu früh ist, bestärkt die vorliegende Studie die Wirksamkeit unserer medikamentösen Therapien und stellt die scharfe Kritik an SSRI in Frage. Eine unbehandelte Depression stellt immer noch das größte Risiko für Suizidalität dar, Achtsamkeit gegenüber Nebenwirkungen darf nicht zu fehlenden Therapieangeboten führen.

In der fehlenden Reduktion der Suizidalität in der jugendlichen Gruppe zeigt sich die Komplexität des Phänomens Suizidalität hier besteht dringender Forschungsbedarf!

Alexandra Kleimann

Gibbons RD et al. Suicidal thoughts and behavior with antidepressant treatment: reanalysis of the randomized placebo-controlled studies of fluoxetine and venlafaxine. Arch Gen Psychiatry 2012; 69: 580-7 